\title{
A case of a giant left ventricular pseudoaneurysm
}

\author{
Stephanie Tan MD, Philippe Romeo MD, Konstantin Papas MD, Patrick Garceau MD, \\ Jocelyn Dupuis MD, Josephine Pressacco MD
}

S Tan, P Romeo, K Papas, P Garceau, J Dupuis, J Pressacco. A case of a giant left ventricular pseudoaneurysm. Curr Res Cardiol 2015;2(2):94-96.

Cardiac pseudoaneurysm is a rare but potentially lethal complication of myocardial infarction. Clinical manifestations may be nonspecific or, in some cases, may even be silent. Therefore, high clinical suspicion should be maintained when a patient becomes unstable after a myocardial event. Efficient use of imaging examinations is crucial in providing an accurate

\section{CASE PRESENTATION}

A 74-year-old man was brought to the emergency department at the authors' institution for severe chest pain that began $30 \mathrm{~min}$ before his arrival. His work-up suggested an acute myocardial infarction (MI) of the inferior territory. Cardiac catheterization demonstrated an occlusion of the middle segment of the right coronary artery, which was successfully dilated without any immediate complications. Four hours later, the patient became unstable and complained of pleuritic chest pain.

On transthoracic echocardiography (TTE), the right ventricle was not clearly identified. A large, heterogeneous and polylobulated mass measuring $7 \mathrm{~cm} \times 13 \mathrm{~cm}$ was noted on the right side of the heart (Figure 1A). It was not possible at the time to determine whether the mass was intraventricular or extrapericardial. With contrast injection, there was heterogeneous enhancement of the mass (Figure 1B).

Several hours later, cardiac magnetic resonance imaging (MRI) demonstrated a large pseudoaneurysm on the posteroinferior segment of the heart measuring $7.0 \mathrm{~cm} \times 11.4 \mathrm{~cm} \times 6.0 \mathrm{~cm}$. A narrow collar measuring $8 \mathrm{~mm}$ was identified on the inferolateral portion of the left diagnosis and, thereafter, timely and appropriate treatment. The authors report a case involving a giant left ventricular pseudoaneurysm that required both transthoracic echocardiography and magnetic resonance imaging to reach the correct diagnosis. The pathology, clinical presentation, diagnostic work-up and treatments of cardiac pseudoaneurysms are reviewed.

Key Words: Computing images; Magnetic resonance imaging; Transthoracic echocardiography; Ventricular pseudoaneurysm

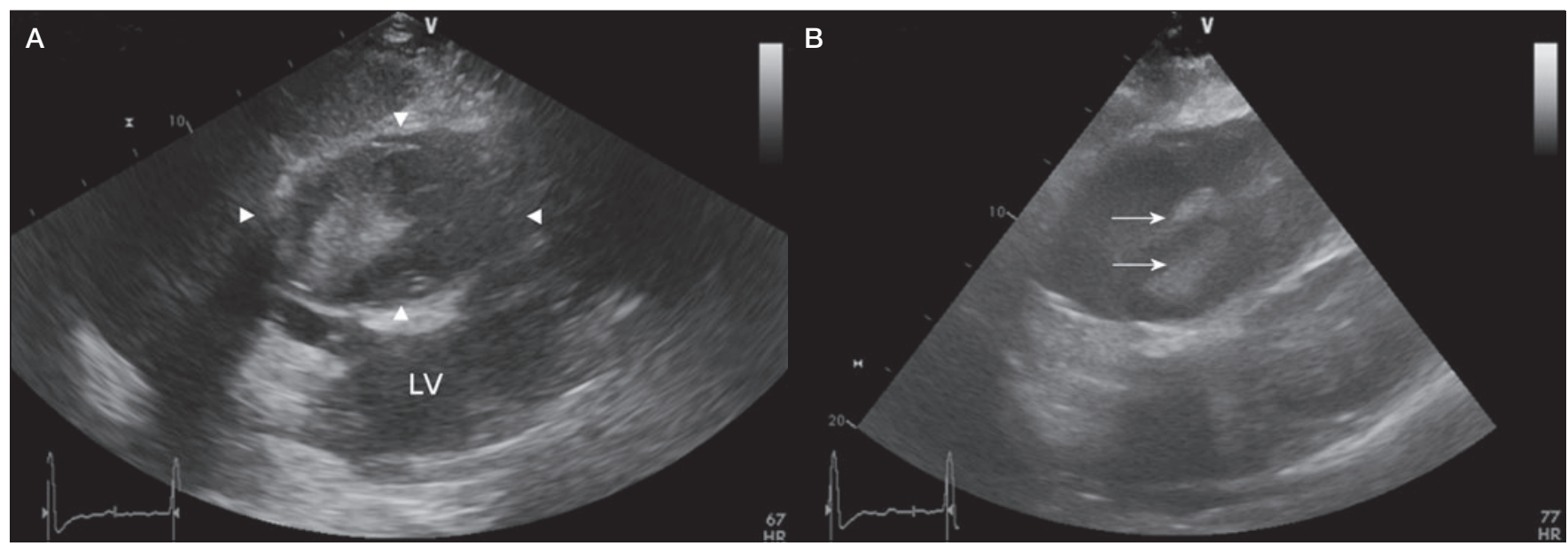

Figure 1) Subcostal images of a transthoracic echocardiography. A The right cardiac chambers are not identified and are compressed by a large heterogeneous structure (arrowheads) representing a large and partially thrombosed pseudoaneurysm. B After contrast injection, the circulating portion (arrows) of the pseudoaneurysm is enhanced. LV Left ventricle

Montreal Heart Institute/University of Montreal, Montreal, Quebec

Correspondence: Dr Stephanie Tan, Montreal Heart Institute, 5000 Belanger Street, Montreal, Quebec H1T 1C8. Telephone 514-376-3330,

e-mail stephanie.tan@umontreal.ca

This open-access article is distributed under the terms of the Creative Commons Attribution Non-Commercial License (CC BY-NC) (http:// creativecommons.org/licenses/by-nc/4.0/), which permits reuse, distribution and reproduction of the article, provided that the original work is properly cited and the reuse is restricted to noncommercial purposes. For commercial reuse, contact support@pulsus.com 


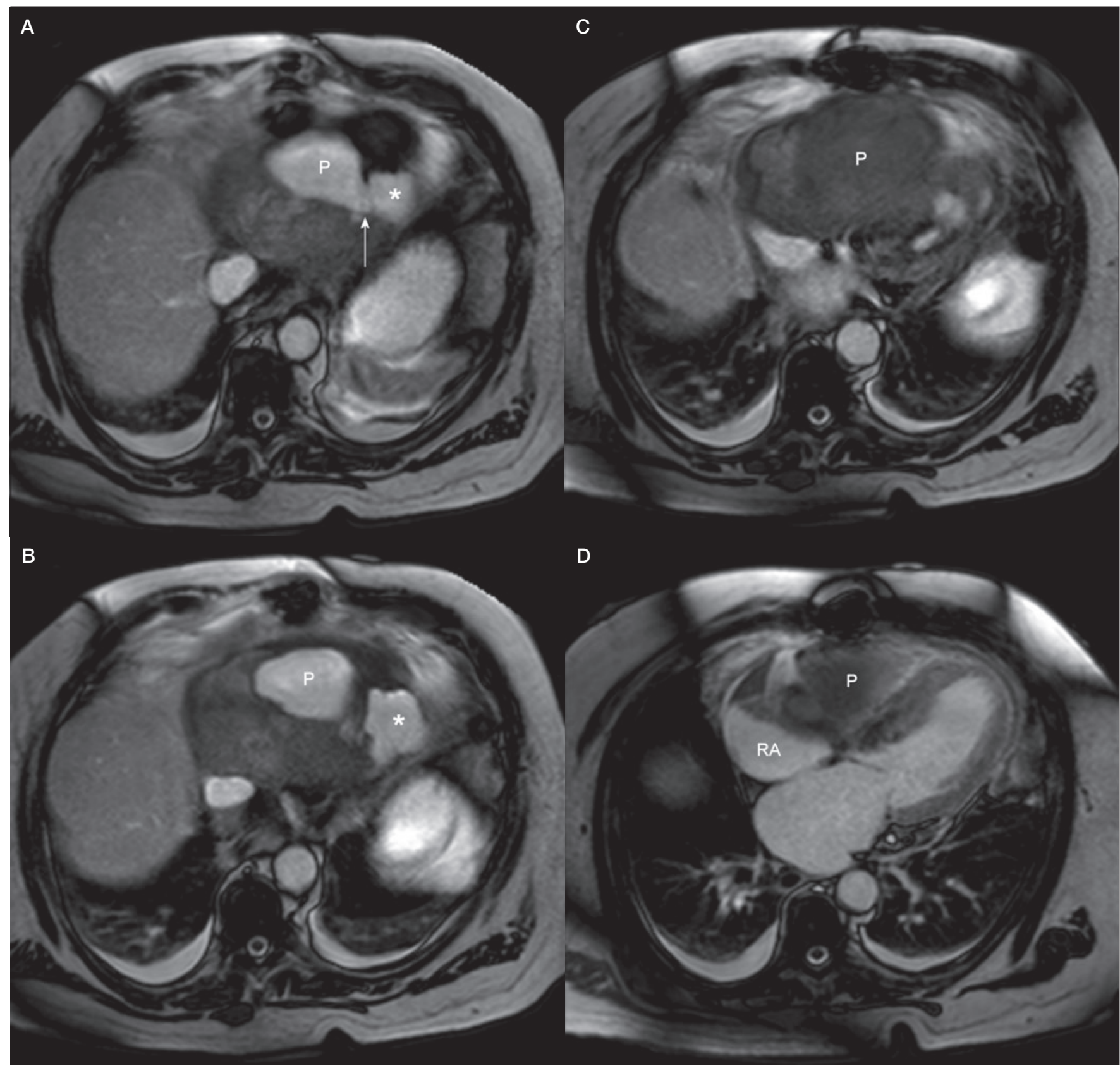

Figure 2) CINE 4 chambers view of cardiac magnetic resonance imaging shows cranial to caudal (A to D) selected images. The giant pseudoaneurysm (P) is thrombosed at an estimated $50 \%$ of its volume and partially compresses the right atria (RA). In panel A, a communication (arrow) between the pseudoaneurysm and the left ventricle (*) is identified

endocardium and the myocardium are damaged. Pseudoaneurysms occur in $4 \%$ of MIs (1). Other less common causes include cardiac surgery, trauma and infection. Typically, pseudoaneurysms are located in the posterior or lateral portion of the left ventricle (2). However, it has been hypothesized that anterior pseudoaneurysm more often lead to rupture and are, therefore, rarely identified (2).

Clinically, patients may present with nonspecific chest pain and dyspnea. However, in nearly $50 \%$ of cases, pseudoaneurysms may remain asymptomatic (3). On heart auscultation, pericardial friction rub and decreased heart sounds may be noted. Electrocardiography will show persistent ST elevation at the site of infarction.

TTE, although rapid and accessible, is diagnostic for left ventricular pseudoaneurysm in only $26 \%$ of cases (2). Occasionally, it may detect a discontinuity of the ventricular wall with blood flow outside of the ventricle. Typically, Doppler will show bidirectional flow in and out of the pseudoaneurysm. Ultrasonography may also demonstrate the narrow neck of the pseudoaneurysm with a ratio of the maximum diameter of the orifice to the maximum internal diameter of the cavity of the sac between 0.25 and 0.50 (4). This is in contrast to true aneurysms, which have ratios $>0.90$.

Conventional angiography, transesophageal echocardiography and cardiac computed tomography are alternative imaging modalities that may provide the diagnosis. However, cardiac MRI is considered to be the best imaging modality to assess pseudoaneurym if available and if the patient's condition allows it (4). It offers better visualization and measurements of the pseudoaneurysm and its neck. As can be expected, there are also findings consistent with MI, including late gadolinium enhancement and akinesia or dyskinesia 


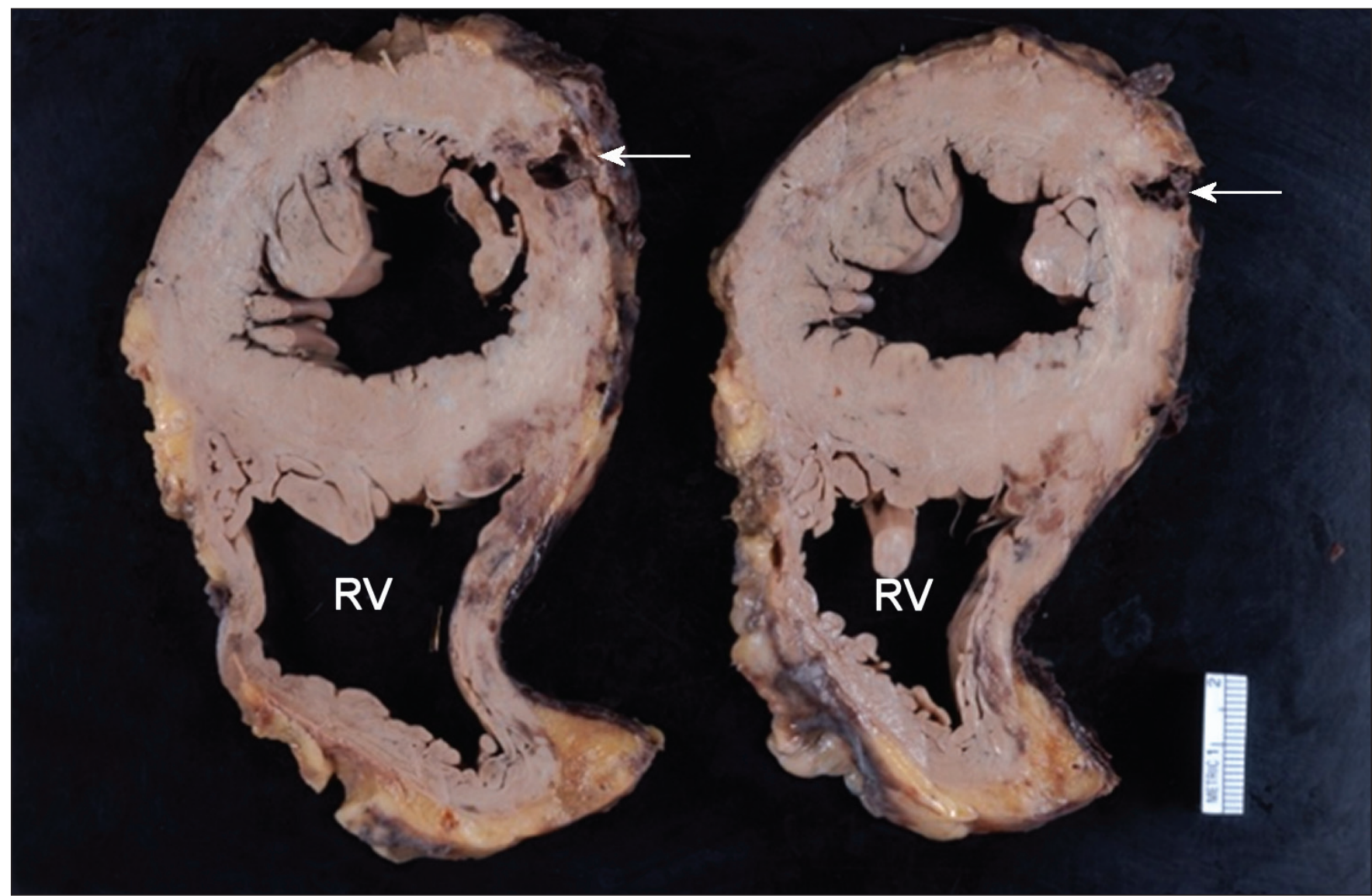

Figure 3) A rupture point was identified on the infero-lateral wall of the left ventricle (arrow). There is slight discoloration on each side of the rupture point, suggesting an acute myocardial infarction. The posterior wall of the right ventricle (RV) is slightly misshapen due to the mass effect produced by the pseudoaneurysm

in the territory of cardiac necrosis. The presence of thrombus in the pseudoaneurysm, valve function and ventricular ejection fraction are more easily evaluated with MRI and provide additional information that may guide clinical decisions.

Because this entity is relatively uncommon, there have been no randomized controlled trials for the appropriate management of left ventricular pseudoaneurysms. If untreated, they are associated with a $30 \%$ to $45 \%$ risk of rupture and represent $23 \%$ of fatal complications of MI (1). Several cases of chronic ventricular pseudoaneurysms and some cases of conservative treatment of pseudoneurysms with good outcome have been reported $(3,5)$. However, a review of recent literature suggests that more aggressive treatment should be considered for large or acute ventricular pseudoaneurysm appearing within three months of MI, especially if located in the anterior or lateral wall, and for patients with arrhythmia or recurrent embolisms due to the pseudoaneurysm $(3,6)$. Surgical intervention aims to restore vascularization at sites of ischemia and to reduce the size

\section{REFERENCES}

1. Zoffoli G, Mangino D, Venturini A, et al. Diagnosing left ventricular aneurysm from pseudo-aneurysm: A case report and a review in literature. J Cardiothorac Surg 2009;4:11.

2. Frances C, Romero D, Grady G. Left ventricular pseudoanueurysm. J Am Coll Cardiol 1998;32:557-61.

3. Yeo TC, Malouf JF, Oh JK, et al. Clinical profile and outcome in 52 patients with cardiac pseudoaneurysm. Ann Intern Med 1998;128:299-305. of the left ventricle. An emergent and less invasive treatment option now includes percutaneous closure of the pseudoaneurysm, which may be more appropriate for high-risk surgical candidates (7).

\section{CONCLUSION}

Ventricular pseudoaneurysms are rare and difficult to diagnose clinically because symptoms are often nonspecific or absent. The interpretation of images provided by TTE can be confusing, especially in atypical cases such as in our patient with a large pseudoaneurysm. Clinical suspicion must remain high in this setting, particularly in post-MI patients. Further imaging, ideally cardiac MRI, can provide the correct diagnosis and additional information that may aid in the selection of the appropriate treatment.

DISCLOSURES: The authors have no conflict of interests to disclose.

4. Laxman D, Rabindra T, Ridhi A, et al. Unruptured left ventricular pseudoaneurysm following inferior wall myocardial infarction. Cardiol J 2012;19:539-42.

5. Hirt LS. Chronic left ventricular pseudo-aneurysm after posterior myocardial infarction. BMJ Case Rep 2012; pii: bcr2012006533.

6. Hulten EA, Blankstein R. Pseudoaneurysms of the heart. Circulation 2012;125:1920-5.

7. Dudiy Y, Jelnin V, Einhorn BN, et al. Percutaneous closure of left ventricular pseudoaneurysm. Circ Cardiovasc Interv 2011;4:322-6. 